\title{
Diagnostic Value of the CD103+CD4+/CD4+ Ratio to Differentiate Sarcoidosis from Other Causes of Lymphocytic Alveolitis
}

\author{
Lisa Bretagne $^{a} \quad$ Ibrahima-Dina Diatta $^{b} \quad$ Mohamed Faouzi ${ }^{b} \quad$ Antoine Nobile $^{c}$ \\ Massimo Bongiovannic ${ }^{\mathrm{C}}$ Laurent P. Nicod $^{\mathrm{d}}$ Romain Lazor $^{\mathrm{d}} \mathrm{e}$ \\ ${ }^{a}$ Lausanne University School of Medicine, and ${ }^{b}$ Institute of Social and Preventive Medicine, ${ }^{\mathrm{C}}$ Institute of Pathology

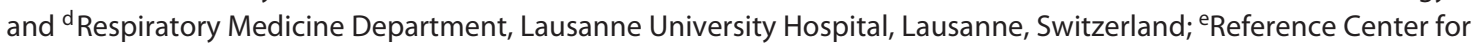 \\ Rare Pulmonary Diseases, Lyon, France
}

\section{Key Words}

Sarcoidosis · Bronchoalveolar lavage · aE integrins · Lung diseases · Interstitial · CD4-positive T lymphocytes · Flow cytometry $\cdot$ Diagnosis

\footnotetext{
Abstract

Background: The CD103 integrin is present on CD4+ lymphocytes of the bronchial mucosa, but not on peripheral blood CD4+ lymphocytes. It has been hypothesized that CD4+ lymphocytes in pulmonary sarcoidosis originate from redistribution from the peripheral blood to the lung, and therefore do not bear the CD103 integrin. Some data suggest that a low CD103+ percentage among bronchoalveolar lavage fluid (BALF) CD4+ lymphocytes discriminates between sarcoidosis and other diagnoses. Objective: To determine the diagnostic value of BALF CD103+ to identify sarcoidosis among other causes of alveolar lymphocytosis in a large retrospective case series. Methods: Among 391 consecutive bronchoalveolar lavages performed at our institution and analyzed by flow cytometry, we identified 207 cases, which were grouped into nine diagnostic categories: sarcoidosis, tuberculosis, non-tuberculous infections, hypersensitivity pneumonitis, non-specific interstitial pneumonia, organizing pneumonia, drug-induced lung diseases,
}

other interstitial lung diseases (ILDs), and other diagnoses. To assess the discriminative value of the CD103+CD4+/ $\mathrm{CD} 4+$ ratio to distinguish sarcoidosis from other entities, areas under ROC curves (AUC) were calculated. Results: Sarcoidosis patients $(n=53)$ had significantly lower CD103+CD4+/CD4+ ratios than patients in other diagnostic categories. The AUC was $62 \%$ for sarcoidosis compared to all other diagnoses, and $69 \%$ for sarcoidosis compared to other ILDs. When combining CD103+CD4+/CD4+ and CD4+/ CD8+ ratios, the AUC increased to 76 and $78 \%$, respectively. When applying previously published cut-offs to our population, the AUC varied between 54 and $73 \%$. Conclusions: The CD103+CD4+/CD4+ ratio does not accurately discriminate between sarcoidosis and other causes of lymphocytic alveolitis, neither alone nor in combination with the CD4+/CD8+ ratio, and is not a powerful marker for the diagnosis of sarcoidosis.

(c) 2016 S. Karger AG, Basel

\section{Introduction}

Sarcoidosis is a chronic multisystemic disease of unknown origin characterized by granulomatous inflammation in various organs, which affects the lungs in $90 \%$

\section{KARGER}

E-Mail karger@karger.com

www.karger.com/res
(C) 2016 S. Karger AG, Basel

0025-7931/16/0916-0486\$39.50/0
Dr. Romain Lazo

Service de pneumologie, Policlinique médicale universitaire

PMU BU44/07.2111, Rue du Bugnon 44

$\mathrm{CH}-1011$ Lausanne (Switzerland)

E-Mail romain.lazor@chuv.ch 
of cases [1]. The diagnosis of pulmonary sarcoidosis is based on clinical symptoms, chest imaging, cytologic analysis of bronchoalveolar lavage fluid (BALF), and histopathology obtained by either bronchial biopsy, transbronchial lung biopsy, transbronchial needle aspiration of mediastinal lymph nodes, or rarely surgical lung biopsy [2-4].

Pulmonary sarcoidosis is characterized by increased $\mathrm{T}$ cells in BALF compared to healthy subjects [5]. These T cells are predominantly $\mathrm{CD} 4+$, with a $\mathrm{CD} 4+/ \mathrm{CD} 8+$ ratio of typically $3: 1$ to $5: 1$, as compared to $2: 1$ in healthy subjects [6]. However, the diagnostic value of BALF in sarcoidosis is limited by the lack of a specific and sensitive marker. BALF lymphocytosis is not specific to sarcoidosis, and the use of the CD4+/CD8+ ratio is controversial, except when it is $>3.5$ and only if the disease is active [7].

Integrin $\alpha E \beta 7$ (CD103) is an adhesion molecule expressed on $95 \%$ of intraepithelial lymphocytes within mucosal tissues, but on $<2 \%$ of circulating peripheral blood lymphocytes [8-12]. A distinct expression pattern of CD103 on BALF CD4+ lymphocytes has been observed in various interstitial lung diseases (ILDs), including sarcoidosis $[12,13]$. It has been hypothesized that most CD4+ lymphocytes in pulmonary sarcoidosis do not originate from bronchial mucosa-associated lymphoid tissue, but from redistribution from the peripheral blood and compartmentalization to the lung [14]. As the peripheral blood CD4+ lymphocytes do not express CD103, differential expression of this marker on BALF CD4+ lymphocytes could serve as a diagnostic marker of sarcoidosis as compared to other ILDs. Several retrospective studies have explored this issue [13, 15-18]. Kolopp-Sarda et al. [15] found that a high $\mathrm{CD} 4+/ \mathrm{CD} 8+$ ratio combined with a low $\mathrm{CD} 103+\mathrm{CD} 4+/ \mathrm{CD} 4+$ ratio allowed to distinguish sarcoidosis from other ILDs with a $96 \%$ sensitivity. Similarly, Mota et al. [17] found a $98 \%$ sensitivity. However, two other studies showed a lower performance of the CD103+CD4+/CD4+ ratio as a diagnostic marker for sarcoidosis $[16,18]$. The goal of the present study was to further investigate the value of the $\mathrm{CD} 103+\mathrm{CD} 4+/ \mathrm{CD} 4+$ ratio to differentiate sarcoidosis from other causes of lymphocytic alveolitis in a large retrospective case series.

\section{Materials and Methods}

\section{Case Selection}

Since 2006, flow cytometry has been routinely performed at the Institute of Pathology of Lausanne University Hospital on all BALF samples with $>20 \%$ lymphocytes on differential cell count, and on samples with $<20 \%$ lymphocytes in case of immunosup- pression or hematological malignancies. For the purpose of this study, flow cytometry results of BALF from Lausanne University Hospital were retrospectively reviewed. Cases with mention of poor BALF quality were discarded. For the remaining cases, electronic medical records were reviewed for diagnostic information, using the pre-defined diagnostic criteria mentioned below.

The diagnosis of sarcoidosis required compatible clinical and imaging features and the presence of non-caseating granuloma on tissue biopsy or transbronchial needle aspiration cytology of mediastinal lymph nodes. Typical stage 1 sarcoidosis occurring in the context of Löfgren syndrome without biopsy or cytology was also accepted. The diagnosis of pulmonary tuberculosis required the presence of acid-fast bacilli, positive polymerase chain reaction and/or positive cultures for Mycobacterium tuberculosis on sputum, bronchial aspiration, or BALF. A diagnosis of infection other than tuberculosis required either identification of a virus or bacterium on sputum, bronchial aspiration or BALF by polymerase chain reaction and/or positive cultures, or marked clinical improvement with antibiotic therapy. Hypersensitivity pneumonitis (HP) was diagnosed according to the criteria of Schuyler and Cormier [19]. The diagnosis of drug-induced pneumonia was based on a history of exposure to a drug with known pulmonary toxicity, a temporal relationship between exposure and onset of symptoms, and improvement after drug withdrawal. Organising pneumonia (OP) was diagnosed on the basis of compatible clinical and imaging features, and presence of buds of granulation tissue on transbronchial lung biopsy without any feature suggesting another diagnosis. The diagnosis of OP was also accepted in the absence of biopsy if radiation therapy for breast cancer, a classical cause of OP, had occurred within the past year. Non-specific interstitial pneumonia (NSIP) was diagnosed on surgical lung biopsy. A diagnosis of NSIP pattern was also accepted if the clinical and imaging findings were highly suggestive even in the absence of surgical lung biopsy. Cases not meeting the above diagnostic criteria were excluded.

Included cases were grouped into nine diagnostic categories: sarcoidosis, tuberculosis, non-tuberculous infections, HP, NSIP, $\mathrm{OP}$, drug-induced lung diseases, other ILDs, and other diagnoses. As some categories had a small number of cases, cases were further grouped into four larger categories: sarcoidosis, infections (including tuberculosis and non-tuberculous infections), ILDs (including HP, NSIP, OP, drug-induced lung diseases, and other ILDs), and other diagnoses. Statistical analysis was performed for both the nine- and four-category distributions.

\section{Bronchoalveolar Lavage and Flow Cytometry}

BALF was collected in sterile bottles during regular diagnostic work-up and filtered through gauze. Ten milliliters of the sample were cytocentrifuged, then smeared and stained with May-Grünwald Giemsa, modified Papanicolaou, Prussian Blue, Gram-Weigert and Ziehl-Neelsen as needed. For differential cell count, a sample corresponding to 250,000-300,000 cells was passed through a multipore filter. After staining with modified Papanicolaou, a minimum of $2 \times 100$ cells were counted.

For flow cytometry, $10-30 \mathrm{ml}$ of native sample were centrifuged, decanted and resuspended in $\mathrm{NH}_{4} \mathrm{Cl}$ or Roswell Park Memorial Institute medium several times until reaching a cellularity of $2 \times 10^{6}$. The cells were stained with fluorochrome-labeled monoclonal antibodies (Dako Denmark A/S, Glostrup, Denmark) for CD45 (FITC, clone T29/33), CD4 (RPE, clone MT310), CD8 
Fig. 1. Graph of the case selection process showing the number of included and excluded cases and the number of cases in each category. $\mathrm{BAL}=$ Bronchoalveolar lavage.

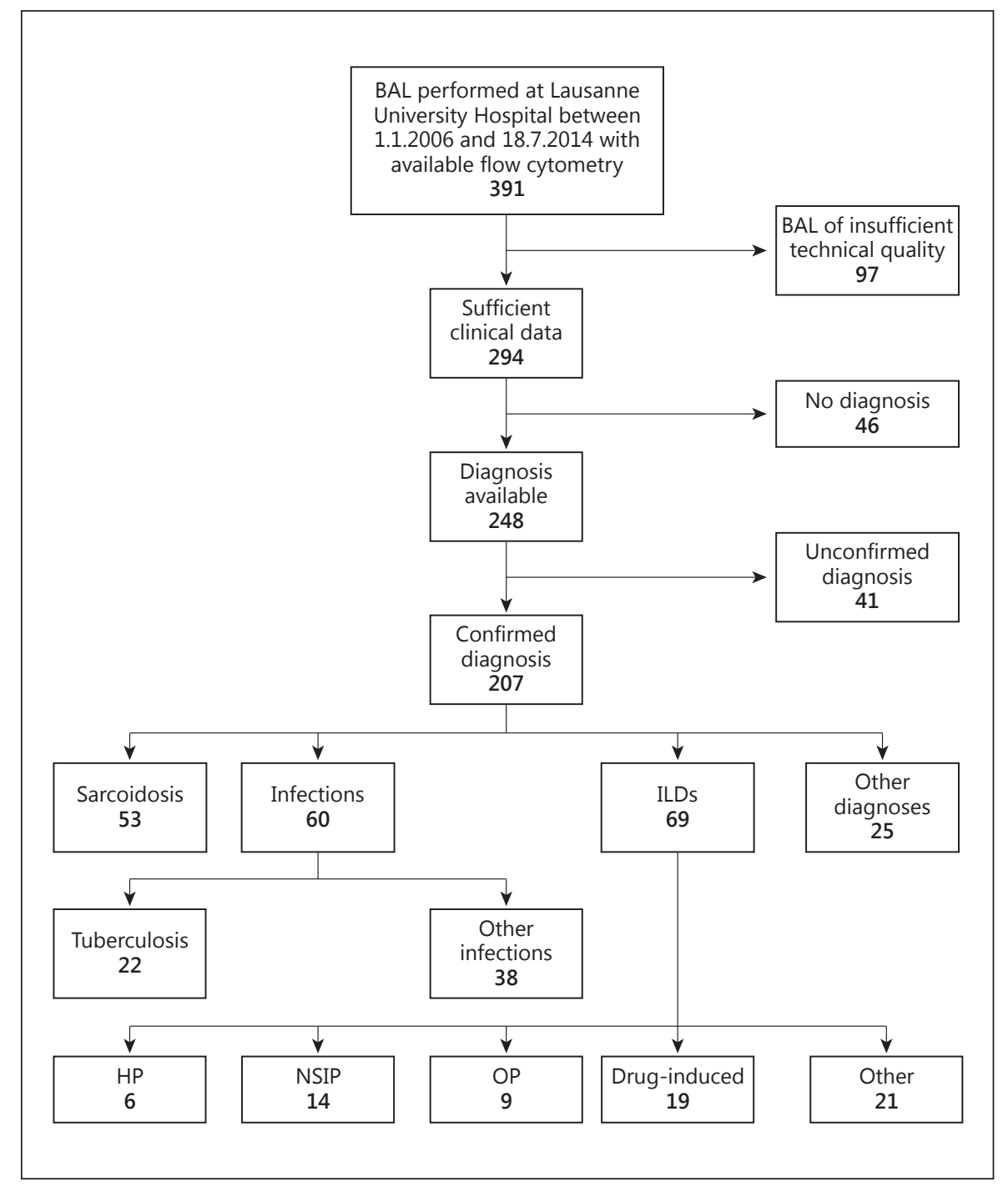

(FITC, clone DK25), and CD103 (FITC, clone Ber-ACT8) following a standard protocol, and five-color flow cytometric analysis was performed (Navios Beckman Coulter flow cytometer).

\section{Statistical Analysis}

Data analyses were performed using Stata Statistical Software, release 13 (StataCorp LP, College Station, Tex., USA). Data were summarized as median and interquartile range (IQR) for continuous variables, and as number and percentage for categorical variables. Group comparison was performed using the Wilcoxon test, and the Kruskal-Wallis test when comparing more than two groups. To assess the discriminative power of the cellular marker ratios $\mathrm{CD} 103+\mathrm{CD} 4+/ \mathrm{CD} 4+$ and $\mathrm{CD} 4+/ \mathrm{CD} 8+$ or their combination to distinguish sarcoidosis from other diagnoses, a ROC analysis was used. Sensitivity, specificity and precision were reported, and the best threshold for optimal sensitivity and specificity was calculated. The study was approved by the ethics committee of the canton of Vaud (CER-VD 241/13).

\section{Results}

\section{Study Population}

The case selection process is summarized in figure 1 . Three hundred and ninety-one consecutive flow cytometries of BALF performed at Lausanne University Hospital between January 1st, 2006 and July 18th, 2014 were reviewed. Ninety-seven cases were excluded due to insufficient technical quality of the BALF sample. Another 87 cases were excluded because the final diagnosis was unclear or unavailable $(n=46)$, or because pre-defined diagnostic criteria were not met $(n=41)$. These 41 cases were the following: suspicion of sarcoidosis without histological diagnosis $(\mathrm{n}=13)$, suspicion of tuberculosis without microbiological evidence of $M$. tuberculosis in- 
Table 1. Demographic and BALF characteristics of the study population

\begin{tabular}{|c|c|c|c|c|c|c|c|c|c|}
\hline Tuberculosis & 22 & 37 & 31 & 39 & 18 & 3 & 7 & 0 & 1 \\
\hline Other infections & 38 & 61 & 21 & 43 & 28 & 9 & 20 & 0 & 2 \\
\hline HP & 6 & 36 & 37 & 49 & 27 & 24 & 25 & 2 & 2 \\
\hline Drug-induced pneumonia & 19 & 71 & 18 & 55 & 34 & 4 & 6 & 1 & 2 \\
\hline Other ILDs & 21 & 59 & 23 & 51 & 41 & 7 & 7 & 1 & 3 \\
\hline Other diagnoses & 25 & 57 & 19 & 45 & 29 & 4 & 9 & 1 & 1 \\
\hline Total & 207 & 56 & 31 & 46 & 29 & 4 & 8 & 1 & 2 \\
\hline
\end{tabular}

fection ( $\mathrm{n}=7$ ), suspicion of non-tuberculous infection without sufficient evidence to support this diagnosis $(\mathrm{n}=$ 10 ), and suspicion of OP without histological proof ( $\mathrm{n}=$ 11).

The remaining 207 cases fulfilling the pre-defined diagnostic criteria were included. Their distribution into nine diagnostic categories is shown in figure 1. Sarcoidosis $(n=53)$ was diagnosed by tissue biopsy $(n=22)$, transbronchial needle aspiration of mediastinal lymph nodes $(n=25)$, or typical Löfgren syndrome $(n=6)$. Nontuberculous pulmonary infection $(\mathrm{n}=38)$ was diagnosed by identification of a virus or bacterium $(n=23)$ or marked clinical improvement with antibiotic therapy $(\mathrm{n}=15)$. OP $(\mathrm{n}=9)$ was diagnosed by transbronchial biopsy $(n=5)$ or a history of radiation therapy to the breast $(\mathrm{n}=4)$. NSIP $(\mathrm{n}=14)$ was diagnosed by surgical lung biopsy $(\mathrm{n}=2)$ or highly suggestive clinical and imaging features $(\mathrm{n}=12)$.

The main characteristics of the study population are presented in table 1 . The median age was 56 years (IQR = 31 ), and 100 patients (48\%) were women. Patients with sarcoidosis, HP and tuberculosis were significantly younger than patients of other diagnostic categories $(\mathrm{p}=$ $0.0001)$. BALF neutrophils were significantly lower in sarcoidosis and higher in HP and non-tuberculous infections $(\mathrm{p}=0.0001)$. No significant differences were found between categories for BALF lymphocyte count.

The 53 patients with sarcoidosis had a median age of 46 years $(\mathrm{IQR}=20)$, and $23(43 \%)$ were women. Their main characteristics are presented in table 2 . At the time of BALF sampling, only 5 were under therapy for sarcoidosis. The median follow-up duration was 2.3 years $(\mathrm{IQR}=3.4)$.

BALF CD103+CD4+/CD4+ Ratio in Sarcoidosis

\section{Analysis of Flow Cytometry in the Nine Diagnostic Categories}

The results of flow cytometry in the nine-category distribution are presented in table 3 . The median value of the CD4+/CD8+ ratio was significantly higher in sarcoidosis compared to all other diagnoses, except for tuberculosis (fig. 2a). ROC curve analysis to discriminate between sarcoidosis and all other diagnoses showed an area under the ROC curve (AUC) of 79\% (fig. 3a). The best cut-off value of CD4+/CD8+ ratio for an optimal specificity and sensitivity was 3.4 .

The $\mathrm{CD} 103+\mathrm{CD} 4+/ \mathrm{CD} 4+$ ratio was significantly lower in sarcoidosis compared to NSIP, HP and other ILDs (table 3, fig. 2b). However, its discriminative power to distinguish between sarcoidosis and all other diagnoses was low, as calculated by the ROC curve analysis (AUC = $62 \%$ ) (fig. 3b). The combination of $\mathrm{CD} 4+/ \mathrm{CD} 8+$ and $\mathrm{CD} 103+\mathrm{CD} 4+/ \mathrm{CD} 4+$ ratios resulted in an AUC of $76 \%$ (fig. 3c), which was lower than the AUC of the CD4+/ CD8+ ratio alone.

\section{Analysis of Flow Cytometry in the Four Diagnostic Categories}

The data were also analyzed after grouping cases into four large categories: sarcoidosis, all infections, all ILDs, and other diagnoses (table 4). The CD4+/CD8+ ratio was significantly higher in sarcoidosis compared to each other category individually (fig. 4a). The only significant difference in the CD103+CD4+/CD4+ ratio was between sarcoidosis and ILDs (fig. 4b), with a higher ratio in ILDs (0.14 vs. $0.08, \mathrm{p}<0.001)$.

Discrimination between sarcoidosis and all ILDs based on the CD4+/CD8+ ratio level had an AUC of 
Table 2. Clinical characteristics of the sarcoidosis population $(n=53)$

\begin{tabular}{|c|c|c|c|c|c|c|c|c|}
\hline & \multicolumn{4}{|c|}{ At diagnosis } & \multicolumn{4}{|c|}{ At last visit } \\
\hline & $\mathrm{n}$ & $\%$ & median & IQR & $\mathrm{n}$ & $\%$ & median & IQR \\
\hline $\mathrm{FEV}_{1}, \%$ predicted & 40 & & 89 & 19 & 35 & & 94 & 18 \\
\hline FVC, \% predicted & 43 & & 102 & 23 & 38 & & 105 & 23 \\
\hline TLC, \% predicted & 39 & & 91 & 17 & 32 & & 93 & 16 \\
\hline $\mathrm{RV}, \%$ predicted & 34 & & 73 & 51 & 27 & & 73 & 27 \\
\hline DLCO, $\%$ predicted & 42 & & 81 & 18 & 35 & & 84 & 27 \\
\hline Normal chest X-ray, \% & 53 & 2 & & & 35 & 28 & & \\
\hline Radiographic stage 1, \% & 53 & 53 & & & 35 & 15 & & \\
\hline Radiographic stage 2, \% & 53 & 40 & & & 35 & 15 & & \\
\hline Radiographic stage 3, \% & 53 & 2 & & & 35 & 6 & & \\
\hline Radiographic stage $4, \%$ & 53 & 4 & & & 35 & 2 & & \\
\hline Phenotype class $1^{*}$ & & & & & 41 & 13 & & \\
\hline Phenotype class $2^{*}$ & & & & & 41 & 9 & & \\
\hline Phenotype class $3^{*}$ & & & & & 41 & 13 & & \\
\hline Phenotype class $4^{*}$ & & & & & 41 & 25 & & \\
\hline Phenotype class $5^{*}$ & & & & & 41 & 2 & & \\
\hline Phenotype class $6^{*}$ & & & & & 41 & 15 & & \\
\hline Eye involvement, \% & 41 & 15 & & & & & & \\
\hline Skin involvement, \% & 41 & 15 & & & & & & \\
\hline Heart involvement, \% & 41 & 4 & & & & & & \\
\hline CNS involvement, \% & 41 & 2 & & & & & & \\
\hline Stomach involvement, \% & 41 & 2 & & & & & & \\
\hline Liver involvement, $\%$ & 41 & 9 & & & & & & \\
\hline Kidney involvement, \% & 41 & 2 & & & & & & \\
\hline Hypercalcemia, \% & 41 & 6 & & & & & & \\
\hline Hypercalciuria, \% & 41 & 13 & & & & & & \\
\hline Löfgren syndrome, \% & 41 & 21 & & & & & & \\
\hline
\end{tabular}

$\mathrm{CNS}=$ Central nervous system; $\mathrm{FEV}_{1}=$ forced expiratory volume in $1 \mathrm{~s} ; \mathrm{RV}=$ residual volume.

* Sarcoidosis clinical activity classification according to Prasse et al. [20].

Table 3. Flow cytometry for the nine-category distribution

\begin{tabular}{|c|c|c|c|c|c|c|}
\hline \multirow[t]{2}{*}{ Diagnostic category } & \multicolumn{3}{|c|}{$\mathrm{CD} 4+/ \mathrm{CD} 8+$ ratio } & \multicolumn{3}{|c|}{$\mathrm{CD} 103+\mathrm{CD} 4+/ \mathrm{CD} 4+$ ratio } \\
\hline & median & IQR & $\mathrm{p}$ value* & median & IQR & p value* \\
\hline Sarcoidosis & 5.3 & 5.9 & - & 0.08 & 0.15 & - \\
\hline Tuberculosis & 2.3 & 2.5 & 0.052 & 0.09 & 0.11 & 0.488 \\
\hline Other infections & 1.7 & 1.9 & $<0.001$ & 0.09 & 0.13 & 0.242 \\
\hline $\mathrm{HP}$ & 1.8 & 3.5 & $<0.001$ & 0.27 & 0.61 & 0.037 \\
\hline NSIP & 0.6 & 1.4 & $<0.001$ & 0.21 & 0.21 & 0.018 \\
\hline $\mathrm{OP}$ & 2.0 & 2.1 & $<0.001$ & 0.10 & 0.09 & 0.698 \\
\hline Drug-induced pneumonia & 1.3 & 2.9 & 0.044 & 0.15 & 0.16 & 0.060 \\
\hline Other ILDs & 1.6 & 3.6 & 0.011 & 0.14 & 0.19 & 0.006 \\
\hline Other diagnoses & 1.6 & 3.1 & 0.010 & 0.10 & 0.16 & 0.106 \\
\hline Total & 2.4 & 3.6 & & 0.10 & 0.17 & \\
\hline
\end{tabular}

* Compared to sarcoidosis. 
Fig. 2. Box-whisker plots of the $\mathrm{CD} 4+/$ $\mathrm{CD} 8+(\mathbf{a})$ and CD103+CD4+/CD4+ (b) ratios in the nine diagnostic categories.

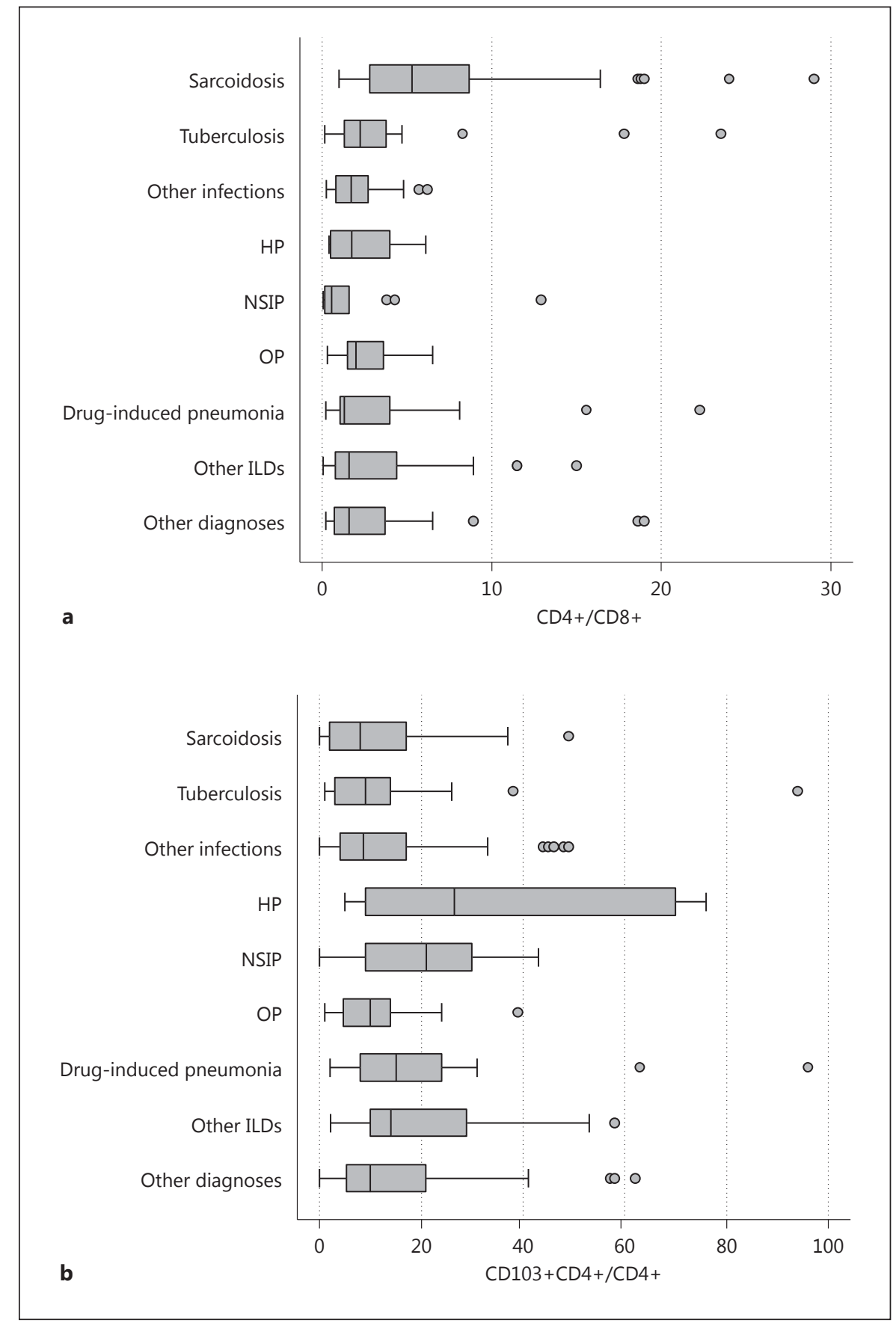

$79 \%$, with an optimal specificity (69\%) and sensitivity (70\%) reached at a threshold of 3.4. However, the calculated AUC for the CD103+CD4+/CD4+ ratio was only $69 \%$. The combination of the CD4+/CD $8+$ and $\mathrm{CD} 103+\mathrm{CD} 4+/ \mathrm{CD} 4+$ ratios resulted in an $\mathrm{AUC}$ of $78 \%$, which was lower than that of the $\mathrm{CD} 4+/ \mathrm{CD} 8+$ ratio alone (data not shown).
Validation of Cut-Off Values from Previous Studies

Four studies analyzing the CD103+CD4+/CD4+ ratio in sarcoidosis, either alone or in combination with the CD4+/CD8+ ratio, have been previously performed [1518]. The cut-off values defined in those studies to discriminate between sarcoidosis and other diagnoses were applied to our study population for comparison (table 5). 

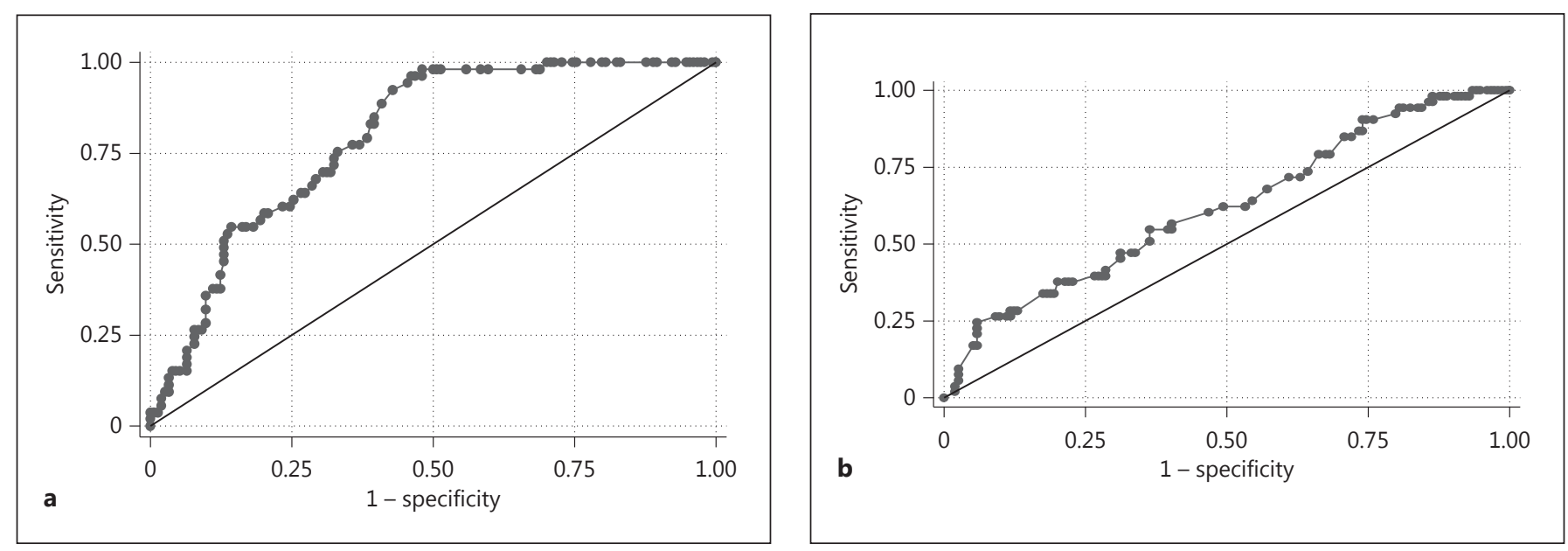

Fig. 3. ROC curves for the CD4+/CD8+ratio(a), the CD103+CD4+/ $\mathrm{CD} 4+$ ratio (b) and their combination (c) to distinguish sarcoidosis from all other diagnoses.

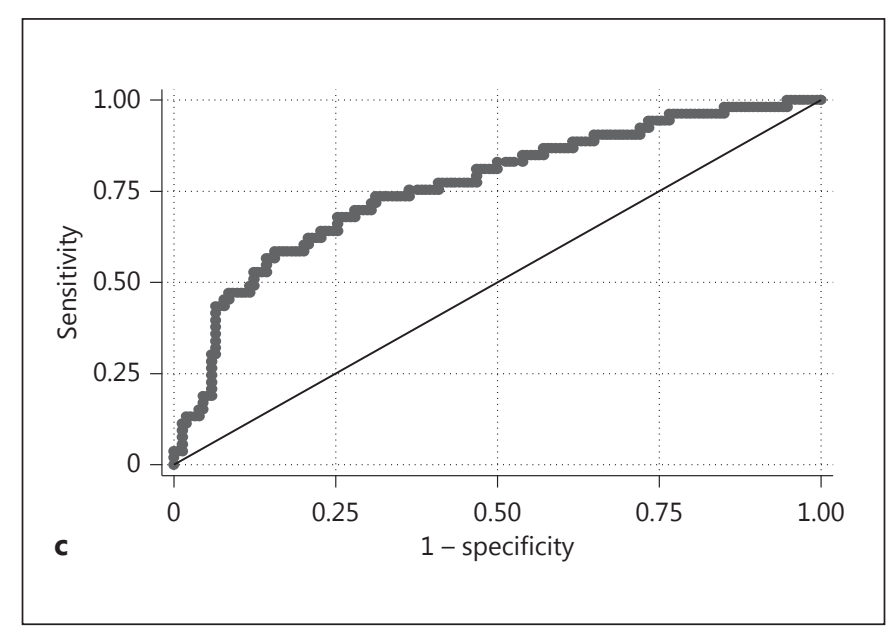

Table 4. Flow cytometry for the four-category distribution

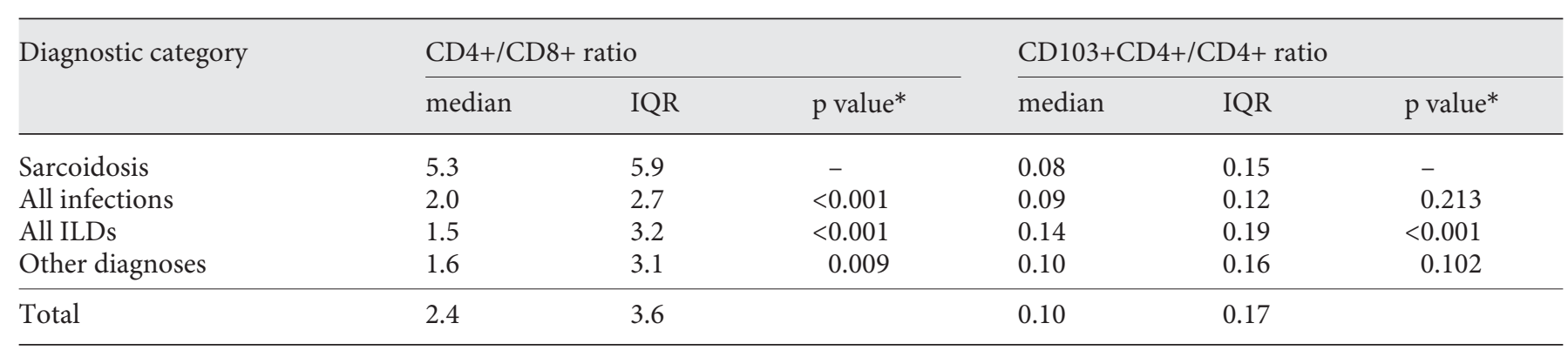

* Compared to sarcoidosis.

Sensitivity varied between 55 and $98 \%$, and specificity between 9 and $86 \%$. The precision of classification varied between 32 and $78 \%$, and the AUC varied between 54 and $73 \%$.
Correlation between the CD103+CD4+/CD4+ Ratio and Other Variables

No correlation was found between the CD103+CD4+/ $\mathrm{CD} 4+$ ratio and the percentage of BALF lymphocytes, 


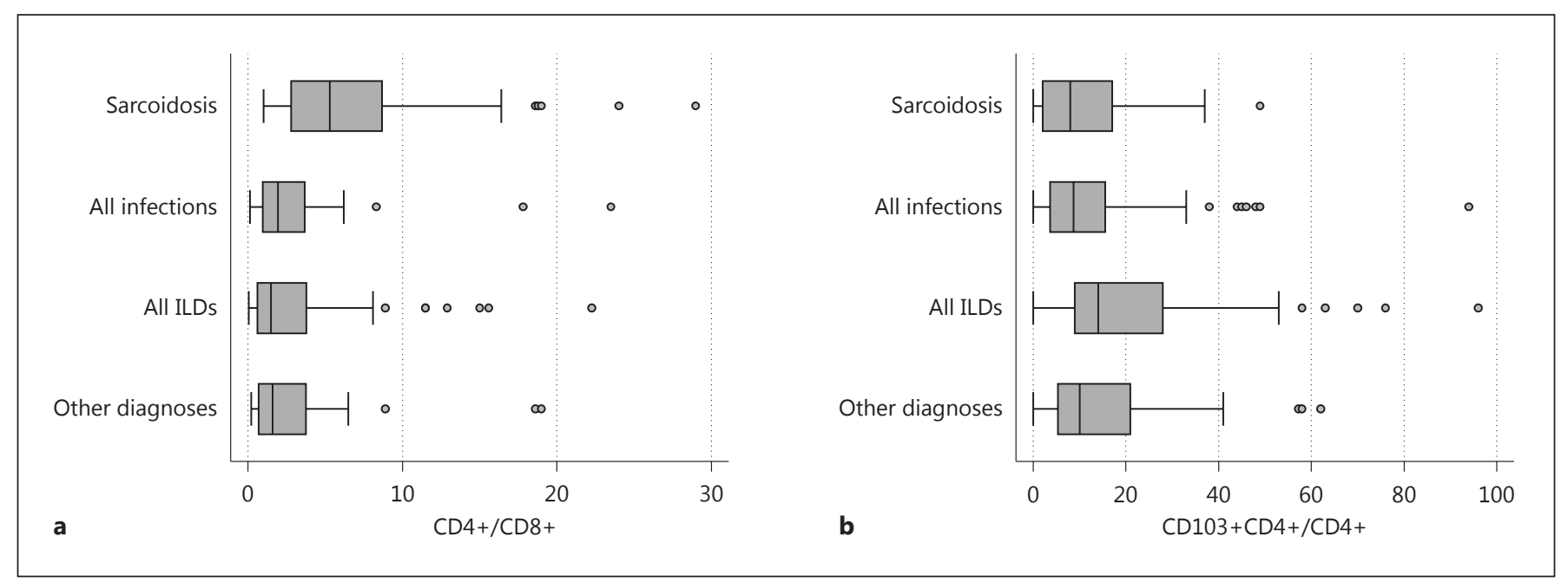

Fig. 4. Box-whisker plots of the CD4+/CD8+ (a) and CD103+CD4+/CD4+ (b) ratios in the four diagnostic categories.

Table 5. Comparison to other studies

\begin{tabular}{|c|c|c|c|c|c|c|c|c|}
\hline Study & Population & Cut-off & $\mathrm{Se}$ & $\mathrm{Sp}$ & $\mathrm{Se}$ & $\mathrm{Sp}$ & $\operatorname{Pr}$ & AUC \\
\hline $\begin{array}{l}\text { Mota et al. } \\
{[17]}\end{array}$ & $\begin{array}{l}\text { total } 86 \text {, } \\
\text { sarcoidosis } 41\end{array}$ & $\mathrm{CD} 103+/ \mathrm{CD} 4+<0.4$ & $81 \%$ & $78 \%$ & $98 \%$ & $9 \%$ & $32 \%$ & $54 \%$ \\
\hline $\begin{array}{l}\text { Hyldgaard } \\
\text { et al. [18] }\end{array}$ & $\begin{array}{l}\text { total } 107 \\
\text { sarcoidosis } 19\end{array}$ & $\begin{array}{l}\mathrm{CD} 4+/ \mathrm{CD} 8+>3.8 \text { and } \\
\mathrm{CD} 103+\mathrm{CD} 4+/ \mathrm{CD} 4+<0.22 \\
\mathrm{CD} 103+\mathrm{CD} 4+/ \mathrm{CD} 4+<0.22\end{array}$ & $\begin{array}{l}42 \% \\
-\end{array}$ & $\begin{array}{l}91 \% \\
-\end{array}$ & $\begin{array}{l}55 \% \\
85 \%\end{array}$ & $\begin{array}{l}86 \% \\
28 \%\end{array}$ & $\begin{array}{l}78 \% \\
43 \%\end{array}$ & $\begin{array}{l}70 \% \\
56 \%\end{array}$ \\
\hline
\end{tabular}

neutrophils and eosinophils, neither in patients with sarcoidosis not in the whole study population (data not shown). Among patients with sarcoidosis, the CD103+CD4+/CD4+ ratio was significantly lower in those with initial radiographic stage 1 (median 0.06, $\mathrm{IQR}=0.15, \mathrm{n}=28$ ) compared to stage 2 (median 0.17, $\mathrm{IQR}=0.17, \mathrm{n}=21, \mathrm{p}=0.02)$. No significant correlation was found between the $\mathrm{CD} 103+\mathrm{CD} 4+/ \mathrm{CD} 4+$ ratio and initial values of forced expiratory volume in $1 \mathrm{~s}$, forced vital capacity (FVC), total lung capacity (TLC), residual volume, and carbon monoxide transfer factor (DLCO). A significant negative correlation was found between the $\mathrm{CD} 103+\mathrm{CD} 4+/ \mathrm{CD} 4+$ ratio and the values at last visit of FVC $(n=37, r=-0.42, p=0.009)$, TLC $(n=31, r=-0.63$, $\mathrm{p}=0.0001)$ and DLCO $(\mathrm{n}=34, \mathrm{r}=-0.37, \mathrm{p}=0.03)$. The occurrence of any extrathoracic organ involvement, the use of therapy at the time of bronchoalveolar lavage, the need for therapy after diagnosis, and the sarcoidosis clin- 
ical activity classification according to Prasse et al. [20] were not correlated with the $\mathrm{CD} 103+\mathrm{CD} 4+/ \mathrm{CD} 4+$ ratio (data not shown).

\section{Discussion}

In the present study, we demonstrated that the BALF lymphocyte CD103+CD4+/CD4+ ratio does not accurately discriminate between sarcoidosis and other causes of lymphocytic alveolitis, neither alone nor in combination with the $\mathrm{CD} 4+/ \mathrm{CD} 8+$ ratio, and is therefore not a relevant marker for the clinical diagnosis of sarcoidosis. Furthermore, the CD103+CD4+/CD4+ ratio does not accurately discriminate between sarcoidosis and other ILDs. When applying the previously published CD103+CD4+/CD4+ ratio cut-offs to our population, we generally found lower sensitivity and specificity than in the original studies.

The CD4+/CD8+ ratio has been previously investigated in order to determine whether it could provide an accurate diagnosis of sarcoidosis without lung biopsy [6, 21-23]. In agreement with these data, we found a significant correlation between sarcoidosis and an elevated CD4+/CD8+ ratio (>3.4 in our study). However, the $\mathrm{AUC}$ of the $\mathrm{CD} 4+/ \mathrm{CD} 8+$ ratio to distinguish sarcoidosis from other causes of lymphocytic alveolitis was only $79 \%$, and it was also $79 \%$ to separate sarcoidosis from other ILDs. Thus, on its own, this marker cannot differentiate between sarcoidosis and other ILDs with enough accuracy to be useful in daily practice.

Four previous studies have investigated the CD103 integrin on CD4+ T lymphocytes in BALF as a putative marker for sarcoidosis [15-18], and several of them have suggested that a high $\mathrm{CD} 4+/ \mathrm{CD} 8+$ ratio combined with a low $\mathrm{CD} 103+\mathrm{CD} 4+/ \mathrm{CD} 4+$ ratio would allow to distinguish sarcoidosis from other causes of alveolar lymphocytosis. Kolopp-Sarda et al. [15] first reported that the combination of a $\mathrm{CD} 4+/ \mathrm{CD} 8+$ ratio $>2.5$ and a CD103+CD4+/CD4+ ratio $<0.31$ had a sensitivity of $96 \%$ to discriminate between sarcoidosis and other causes of lymphocytic alveolitis. They concluded that the combination of both ratios would constitute a good marker for the diagnosis of sarcoidosis. Heron et al. [16] and Mota et al. [17] also reported a good discriminative power of the $\mathrm{CD} 103+\mathrm{CD} 4+/ \mathrm{CD} 4+$ ratio (table 5). However, in another study by Hyldgaard et al. [18], the combination of $\mathrm{CD} 4+/ \mathrm{CD} 8+$ and $\mathrm{CD} 103+\mathrm{CD} 4+/ \mathrm{CD} 4+$ ratios had a sensitivity of only $42 \%$ and a specificity of $91 \%$, which led to conclude that the combination of these ratios was a poor marker for sarcoidosis. Of note, all those studies had small study populations (table 5), and two of them included less than 20 cases with sarcoidosis $[15,18]$.

In our population, the $\mathrm{CD} 103+\mathrm{CD} 4+/ \mathrm{CD} 4+$ ratio had a weak discriminative power for the diagnosis of sarcoidosis, with an AUC of only $62 \%$ to distinguish sarcoidosis from all other causes of lymphocytic alveolitis, and an AUC of $69 \%$ to distinguish sarcoidosis from other ILDs. The use of combined CD4+/CD8+ and $\mathrm{CD} 103+\mathrm{CD} 4+/ \mathrm{CD} 4+$ ratios resulted in modest increases of the AUC to $76 \%$ for sarcoidosis versus all causes of lymphocytic alveolitis, and to $78 \%$ for sarcoidosis versus other ILDs. This remained lower than the AUC of the $\mathrm{CD} 4+/ \mathrm{CD} 8+$ ratio alone (79\%), suggesting that the contribution of the $\mathrm{CD} 103+\mathrm{CD} 4+/ \mathrm{CD} 4+$ ratio itself was low.

We also tested the cut-off values suggested by other studies on our population (table 5). The highest percentage of correctly classified patients (78\%) was achieved by using Hyldgaard et al.'s [18] cut-off, whereas with the one suggested by Mota et al. [17], the percentage of correctly classified patients was very low (32\%). Further analysis of our population using the CD103+CD4+/ $\mathrm{CD} 4+$ ratio alone showed a high sensitivity but a very low specificity, and the precision did not exceed $43 \%$ (table 5). Altogether, this reinforces our own observations suggesting that the $\mathrm{CD} 103+\mathrm{CD} 4+/ \mathrm{CD} 4+$ ratio, either alone or combined with the $\mathrm{CD} 4+/ \mathrm{CD} 8+$ ratio, does not provide sufficient diagnostic precision to identify sarcoidosis among other causes of lymphocytic alveolitis.

The strength of our study was the size of the population $(\mathrm{n}=207)$, which was much larger than in any other study of the CD103+CD4+/CD4+ ratio. The number of sarcoidosis cases $(n=53)$ was also higher than in most other studies and almost equaled the 56 cases reported by Heron et al. [16]. Unlike the other studies, we were able to precisely classify our population into well-defined diagnostic categories and to compare sarcoidosis to each category individually, as well as to broader diagnostic groups. This allowed a more precise analysis of the CD103+CD4+/CD4+ ratio for other diagnoses such as HP, NSIP, infections, and other ILDs.

One limitation of our study is the fact that we included only patients with BALF lymphocytosis $>20 \%$, whereas there have been reports of cases of sarcoidosis with normal or only mildly elevated lymphocyte counts [18, $21,24,25]$, especially in asymptomatic patients [26]. Although this occurs in a minority of sarcoidosis patients, 
this population would have been missed by our study design, and we therefore cannot rule out that the CD 103+CD4+/CD4+ ratio might have greater relevance in sarcoidosis with mild or no BALF lymphocytosis. Another limitation is that not all diagnostic categories were equally represented. For instance, there was only a small number of patients with HP in our population $(n=6)$. This prevented us from directly comparing sarcoidosis to $\mathrm{HP}$ in order to explore the value of the CD103+CD4+/ $\mathrm{CD} 4+$ ratio in separating these two entities. One recent study found a significantly higher CD103+CD4+/CD4+ ratio in HP as compared to sarcoidosis, but no cut-off value was provided to discriminate between these diagnoses [13].

Our observations do not weaken the hypothesis that CD4+ lymphocytes in pulmonary sarcoidosis originate from peripheral blood rather than from resident mucosal lymphoid tissue. Indeed, all studies showed that there is a lower percentage of CD4+ lymphocytes presenting the CD103 integrin in BALF of patients with sarcoidosis as compared to patients with other diseases causing pulmonary lymphocytosis. However, as demonstrated in the present study, the BALF CD103+CD4+/CD4+ ratio does not have enough discriminative power to accurately differentiate between sarcoidosis and other causes of pulmonary lymphocytosis, and cannot replace lung biopsy or lymph node needle aspiration for the diagnosis of pulmonary sarcoidosis. One can hypothesize that CD103 integrin expression may be modified under the effect of lung microenvironment or regulatory $\mathrm{T}$ cells.

In exploratory analyses, we found that the BALF CD103+CD4+/CD4+ ratio was significantly lower in initial radiographic stage 1 sarcoidosis (median 0.06) as compared to stage 2 (median 0.17 ). We also found a significant negative correlation between BALF CD103+CD4+/CD4+ ratio and FVC, TLC and DLCO at last visit. Taken together, these data suggest that the BALF CD103+CD4+/CD4+ ratio may be of interest as a prognostic marker in sarcoidosis. This issue should be addressed in future studies.

\section{Acknowledgements}

The authors acknowledge Nastasia Negro for performing part of the data capture and for preparation of figure 1, and Nathalie Bacco for secretarial assistance.

\section{References}

1 Baughman RP, Lower EE, Gibson K: Pulmonary manifestations of sarcoidosis. Presse Med 2012;41:e289-e302.

-2 King TE Jr: Clinical advances in the diagnosis and therapy of the interstitial lung diseases. Am J Respir Crit Care Med 2005;172:268279.

3 Ryu JH, Olson EJ, Midthun DE, Swensen SJ: Diagnostic approach to the patient with diffuse lung disease. Mayo Clin Proc 2002;77: 1221-1227; quiz 1227.

4 Oikonomou A: Role of imaging in the diagnosis of diffuse and interstitial lung diseases. Curr Opin Pulm Med 2014;20:517-524.

5 Wells AU: The clinical utility of bronchoalveolar lavage in diffuse parenchymal lung disease. Eur Respir Rev 2010;19:237-241.

6 6 Welker L, Jorres RA, Costabel U, Magnussen $\mathrm{H}$ : Predictive value of BAL cell differentials in the diagnosis of interstitial lung diseases. Eur Respir J 2004;24:1000-1006.

7 Costabel U, Bonella F, Ohshimo S, Guzman J: Diagnostic modalities in sarcoidosis: BAL, EBUS, and PET. Semin Respir Crit Care Med 2010;31:404-408.
8 Cerf-Bensussan N, Jarry A, Brousse N, Lisowska-Grospierre B, Guy-Grand D, Griscelli C: A monoclonal antibody (HML-1) defining a novel membrane molecule present on human intestinal lymphocytes. Eur J Immunol 1987;17:1279-1285.

9 Cepek KL, Shaw SK, Parker CM, Russell GJ, Morrow JS, Rimm DL, Brenner MB: Adhesion between epithelial cells and T lymphocytes mediated by E-cadherin and the alpha $\mathrm{E}$ beta 7 integrin. Nature 1994;372:190-193.

10 Cerf-Bensussan N, Begue B, Gagnon J, Meo T: The human intraepithelial lymphocyte marker HML-1 is an integrin consisting of a beta 7 subunit associated with a distinctive alpha chain. Eur J Immunol 1992;22:273-277.

-11 Parker CM, Cepek KL, Russell GJ, Shaw SK, Posnett DN, Schwarting R, Brenner MB: A family of beta 7 integrins on human mucosal lymphocytes. Proc Natl Acad Sci USA 1992; 89:1924-1928.

12 Lohmeyer J, Friedrich J, Grimminger F, Maus U, Tenter R, Morr H, Velcovsky HG, Seeger W, Rosseau S: Expression of mucosa-related integrin alphaEbeta7 on alveolar T cells in interstitial lung diseases. Clin Exp Immunol 1999; 116:340-346.
13 Couto M, Palmares C, Beltrao M, Neves S, Mota P, Morais A, Delgado L: Integrin alpha E beta 7 (CD103) expression in bronchoalveolar lymphocytes of patients with hypersensitivity pneumonitis. Int Arch Occup Environ Health 2015;88:167-173.

14 Semenzato G: Immunology of interstitial lung diseases: cellular events taking place in the lung of sarcoidosis, hypersensitivity pneumonitis and HIV infection. Eur Respir J 1991;4: 94-102.

15 Kolopp-Sarda MN, Kohler C, De March AK, Bene MC, Faure G: Discriminative immunophenotype of bronchoalveolar lavage CD4 lymphocytes in sarcoidosis. Lab Invest 2000; 80:1065-1069.

16 Heron M, Slieker WA, Zanen P, van Lochem EG, Hooijkaas H, van den Bosch JM, van Velzen-Blad H: Evaluation of CD103 as a cellular marker for the diagnosis of pulmonary sarcoidosis. Clin Immunol 2008;126:338-344.

17 Mota PC, Morais A, Palmares C, Beltrao M, Melo N, Santos AC, Delgado L: Diagnostic value of CD103 expression in bronchoalveolar lymphocytes in sarcoidosis. Respir Med 2012;106:1014-1020. 
18 Hyldgaard C, Kaae S, Riddervold M, Hoffmann HJ, Hilberg O: Value of s-ACE, BAL lymphocytosis, and $\mathrm{CD} 4+/ \mathrm{CD} 8+$ and CD103+CD4+/CD4+ T-cell ratios in diagnosis of sarcoidosis. Eur Respir J 2012;39:10371039.

19 Schuyler M, Cormier Y: The diagnosis of hypersensitivity pneumonitis. Chest 1997;111: 534-536.

20 Prasse A, Katic C, Germann M, Buchwald A, Zissel G, Muller-Quernheim J: Phenotyping sarcoidosis from a pulmonary perspective. Am J Respir Crit Care Med 2008;177:330336.
Kantrow SP, Meyer KC, Kidd P, Raghu G: The CD4/CD8 ratio in BAL fluid is highly variable in sarcoidosis. Eur Respir J 1997;10:27162721.

22 Danila E, Norkuniene J, Jurgauskiene L, Malickaite R: Diagnostic role of BAL fluid CD4/ CD8 ratio in different radiographic and clinical forms of pulmonary sarcoidosis. Clin Respir J 2009;3:214-221.

23 Oda K, Ishimoto H, Yatera K, Yamada S, Nakao H, Ogoshi T, Noguchi S, Yamasaki K, Kawanami T, Mukae H: Relationship between the ratios of CD4/CD8 T-lymphocytes in the bronchoalveolar lavage fluid and lymph nodes in patients with sarcoidosis. Respir Investig 2014;52:179-183.
24 Bacha D, Ayadi-Kaddour A, Ismail O, El Mezni F: Bronchoalveolar lavage impact in sarcoidosis: study of 40 cases. Tunis Med 2009;87:38-42.

25 Danila E, Jurgauskiene L, Norkuniene J, Malickaite R: BAL fluid cells in newly diagnosed pulmonary sarcoidosis with different clinical activity. Ups J Med Sci 2009;114:26-31.

26 Drent M, van Velzen-Blad H, Diamant M, Hoogsteden HC, van den Bosch JM: Relationship between presentation of sarcoidosis and T lymphocyte profile. A study in bronchoalveolar lavage fluid. Chest 1993;104:795-800. 